\title{
ON OXYGEN DIFFUSION IN A SPHERICAL CELL WITH MICHAELIS-MENTEN OXYGEN UPTAKE KINETICS
}

\author{
- P. HiltMANN and P. LORY† \\ Institut für Mathematik, \\ Technische Universität München, \\ D-8000 München 2, West Germany
}

This paper demonstrates that there is one and only one solution to a non-linear singular two-point boundary-value problem which describes oxygen diffusion in a spherical cell. Previous authors have calculated numerical results that differ substantially. Numerical computations using the multiple shooting method support the results of McElwain.

1. Introduction. The non-linear singular boundary-value problem

$$
\begin{gathered}
C^{\prime \prime}+\frac{2}{r} \cdot C^{\prime}=\frac{\alpha \cdot C}{C+K_{\mathrm{m}}} \\
C^{\prime}(0)=0, \quad C^{\prime}(1)=H \cdot(1-C(1))
\end{gathered}
$$

describes the steady-state oxygen diffusion in a spherical cell with MichaelisMenten oxygen uptake kinetics (Lin, 1976; McElwain, 1978). Throughout the paper, primes denote differentiation with respect to $r$. All quantities in (1) and (2) are dimensionless, where the normalized variables $C$ and $r$ represent the oxygen tension and the radial distance. The radius of the cell corresponds to $r=1$, and $\alpha, K_{\mathrm{m}}$ and $H$ are positive constants involving the (constant) diffusion coefficient, maximum reaction rate, Michaelis constant and permeability of the cell membrane.

Numerical calculations have been carried out by Lin (1976) and McElwain (1978). However, their results differ substantially. For this reason, Anderson and Arthurs (1980) re-examined the problem numerically using variational methods. Their solution supported the computations of McElwain.

One might attribute these discrepancies to the fact that the solution of the boundary-value problem in (1) and (2) is possibly not uniquely determined. It is the main purpose of this paper to exclude this possibility. Indeed, the theorem in Section 2 proves that the boundary-value problem has one and

$\dagger$ To whom correspondence should be addressed. 
only one solution in the physically feasible range. Careful numerical calculations using the multiple shooting method (see Stoer and Bulirsch, 1980) are presented in Section 3. They support the results of McElwain (1978).

\section{Existence and Uniqueness of the Solution.}

THEOREM. The boundary value problem in (1) and (2) possesses exactly one solution $C(r)$ such that $C(0)$ is positive. This solution is strictly monotone increasing and satisfies the inequality $C(r)<1$.

Proof. The singular initial value problem

$$
\begin{gathered}
C^{\prime \prime}=f\left(r, C, C^{\prime}\right), \text { with } f\left(r, z_{1}, z_{2}\right)=-\frac{2}{r} \cdot z_{2}+\left\{\begin{array}{cc}
\frac{\alpha \cdot z_{1}}{z_{1}+K_{\mathrm{m}}} & \text { for } z_{1} \geqslant 0 \\
0 & \text { for } z_{1}<0
\end{array}\right. \\
C^{\prime}(0)=0, \quad C(0)=\theta \quad(\theta \geqslant 0),
\end{gathered}
$$

has a unique twice continuously differentiable solution. This follows from the application of Theorem 2.1 of de Hoog and Weiss (1977) to the equivalent first-order system. Let $C(r ; \theta)$ denote this solution, stressing its dependence on the initial value $\theta$. A limiting process $r \rightarrow 0^{+}$shows that

$$
3 C^{\prime \prime}(0 ; \theta)=\frac{\alpha \cdot \theta}{\theta+K_{\mathrm{m}}} \text {. }
$$

In the following, $\theta$ is supposed to be positive. So $C^{\prime \prime}(0 ; \theta)>0$. Assume there exists a zero of $C^{\prime}$ in the interval $0<r \leqslant 1$ and let $\rho$ be the smallest one. Then $C^{\prime}(r ; \theta)>0$ for $0<r<\rho$. Hence $C(\rho ; \theta)>0$ and equation (3) implies $C^{\prime \prime}(\rho ; \theta)>0$. Therefore there exists a positive $\epsilon$ such that $0<\rho-\epsilon$ and $C^{\prime}(\rho-\epsilon ; \theta)<0$. This is a contradiction to the definition of $\rho$. Consequently,

$$
C^{\prime}(r ; \theta)>0 \text { for } 0<r \leqslant 1, \quad \theta>0
$$

and

$$
C(r ; \theta)>0 \text { for } 0 \leqslant r \leqslant 1, \quad \theta>0 .
$$

Applying the same argument to $\mathrm{d}(r)=C\left(r ; \theta_{1}\right)-C\left(r ; \theta_{2}\right)$, the following monotonicity properties may be shown:

$$
\left.\begin{array}{ll}
C^{\prime}\left(r ; \theta_{1}\right)>C^{\prime}\left(r ; \theta_{2}\right) & \text { for } 0<r \leqslant 1 \\
C\left(r ; \theta_{1}\right)>C\left(r ; \theta_{2}\right) & \text { for } 0 \leqslant r \leqslant 1
\end{array}\right\} \text { whenever } \theta_{1}>\theta_{2}>0 .
$$


The function $f$ in (3) satisfies a one-sided Lipschitz condition:

$$
f\left(r, z_{1}, z_{2}\right)-f\left(r, \bar{z}_{1}, \bar{z}_{2}\right) \leqslant \frac{\alpha}{K_{\mathrm{m}}} \cdot\left|z_{1}-\bar{z}_{1}\right| \text { for } z_{2} \geqslant \bar{z}_{2} .
$$

So Theorem 14II of Walter (1970) proves that $C(1 ; \theta)$ and $C^{\prime}(1 ; \theta)$ depend continuously on $\theta$. Because of (8) the (continuous) function

$$
G(\theta)=C^{\prime}(1 ; \theta)-H(1-C(1 ; \theta))
$$

is strictly monotone increasing. Obviously, the initial value problem in (3) and (4) has only the trivial solution for $\theta=0$. Hence $G(0)$ is negative. Because of $(6), C(r ; 1)$ is strictly monotone increasing in $r$. Therefore $C(1 ; 1)>C(0 ; 1)=1$. So on the other hand, $G(1)$ is positive.

Hence there exists a uniquely determined positive solution $\theta^{*}$ of the equation $G(\theta)=0$, and so the boundary value problem in (2) and (3) possesses exactly one solution such that $C(0)$ is positive. Because of $(6)$ this solution $C\left(r ; \theta^{*}\right)$ is strictly monotone increasing. As $C^{\prime}\left(1 ; \theta^{*}\right)$ is positive, the second boundary condition in (2) shows that $C\left(r ; \theta^{*}\right)<1$. For $C>0$ the differential equations (1) and (3) coincide. So the proof of the theorem is complete.

3. Numerical Calculations. The boundary value problem in (1) and (2) with the parameter set corresponding to case (d) in the paper of McElwain (1978) was solved by multiple shooting (routine BOUNDS). This method is described in detail in the textbook of Stoer and Bulirsch (1980). For the integration of the initial value sub-problems four different programs have been used (DIFSY1, RKF4, RKF7, VOAS). These routines control the integration step sizes automatically according to built-in estimators of the truncation error. For a brief description and references to the original articles see Diekhoff et al. (1977). The singularity at $r=0$ was treated using both the technique suggested by de Hoog and Weiss (1977) and a Taylor series method (Stoer and Bulirsch, 1980).

All these calculations (Table I), including runs with Lin's solution as the starting trajectory, produced the same results (to the prescribed level of accuracy). They agree well with McElwain's solution, which is given graphically. The table presents the first six digits resulting from a run with high accuracy (prescribed tolerance for Newton's method: $10^{-8}$; for the integrator: $\left.10^{-10}\right)$.

Remark. A similar boundary value problem (simpler boundary conditions at $r=1$ than in (2)) has been computed by Keller (1968) using a finite difference method. 
TABLE I

\begin{tabular}{cc}
\multicolumn{2}{c}{ Numerical Results } \\
\hline$r$ & $C$ \\
\hline 0.0 & 0.828483 \\
0.1 & 0.829706 \\
0.2 & 0.833375 \\
0.3 & 0.839490 \\
0.4 & 0.848053 \\
0.5 & 0.859065 \\
0.6 & 0.872528 \\
0.7 & 0.888445 \\
0.8 & 0.906819 \\
0.9 & 0.927651 \\
1.0 & 0.950946 \\
\hline
\end{tabular}

\section{LITERATURE}

Anderson, N. and A. M. Arthurs. 1980. "Complementary Variational Principles for Diffusion Problems with Michaelis-Menten Kinetics." Bull. math. Biol. 42, 131-135.

Diekhoff, H.-J., P. Lory, H. J. Oberle, H.-J. Pesch, P. Rentrop and R. Seydel. 1977. "Comparing Routines for the Numerical Solution of Initial Value Problems of Ordinary Differential Equations in Multiple Shooting." Num. Math. 27, 449-469.

Hoog, F. R. de and R. Weiss. 1977. "The Application of Linear Multistep Methods to Singular Initial Value Problems." Math. Comput. 31, 676-690.

Keller, H. B. 1968. Numerical Methods for Two-point Boundary-value Problems. Waltham: Blaisdell Publishing Company.

Lin, S. H. 1976. "Oxygen Diffusion in a Spherical Cell with Nonlinear Oxygen Uptake Kinetics." J. theor. Biol. 60, 449-457.

McElwain, D. L. S. 1978. "A Re-examination of Oxygen Diffusion in a Spherical Cell with Michaelis-Menten Oxygen Uptake Kinetics." J. theor. Biol. 71, 255-263.

Stoer, J. and R. Bulirsch. 1980. Introduction to Numerical Analysis. New York: SpringerVerlag.

Walter, W. 1970. Differential and Integral Inequalities. New York: Springer-Verlag.

RECEIVED 6-2-82 